\title{
Experiment-based Performance Improvement of State Feedback Control Systems for Single Input Processes
}

\section{Mircea-Bogdan Rădac ${ }^{1}$, Radu-Emil Precup ${ }^{1}$, Emil M. Petriu ${ }^{2}$, Stefan Preitl ${ }^{1}$}

${ }^{1}$ Department of Automation and Applied Informatics, "Politehnica" University of Timisoara, Bd. V. Parvan 2, RO-300223 Timisoara, Romania

E-mail: mircea.radac@aut.upt.ro, radu.precup@aut.upt.ro, stefan.preitl@aut.upt.ro

${ }^{2}$ School of Electrical Engineering and Computer Science, University of Ottawa, 800 King Edward, Ottawa, Ontario, Canada, K1N 6N5

E-mail: petriu@eecs.uottawa.ca

\begin{abstract}
This paper gives an extension to the Iterative Feedback Tuning (IFT) approach that ensures the performance improvement of state feedback control systems for single input processes. IFT employs sensitivity functions and the experiments conducted on the real-world control system in order to provide an efficient way to deal with the nonlinear or ill-defined processes when the model-dependent Linear-Quadratic Regulator (LQR) is not successful. An experimental setup is suggested to implement the real-time iterative calculation of the gradients in the minimization of the LQR's objective function. The experimental results validate the performance of the proposed IFT algorithm in a mechatronics application which deals with the angular position controller for a DC servo system with actuator dead zone and control signal saturation. The results show the reduction of the LQR's objective function for a single input process application.
\end{abstract}

Keywords: implementation; Iterative Feedback Tuning; mechatronics; state feedback control; real-time experiments

\section{Introduction}

The improvement and optimisation of control system (CS) performance is normally obtained by minimizing objective functions (OFs) with several expressions [1]-[9] including integral quadratic performance indices. This also provides a convenient way to deal with the degrees of freedom associated with the pole placement design of Multi Input-Multi Output (MIMO) systems. 
The Linear-Quadratic Regulator (LQR) approach, which is frequently used for the tuning of the optimal state feedback CSs, can actually be used only when linearized or linear models of the process and the knowledge on all state variables available for feedback are assumed. Alternatively, the Iterative Feedback Tuning (IFT) [10]-[12] offers a direct data-based offline-adaptive controller tuning strategy. IFT solves the problem by a gradient-based minimisation of the OF using data collected from the real-world CS. Attractive applications of IFT reported in the literature are chemical process control [13], servo drive control [14], [15], nonlinear process control [16]-[18], on-line IFT control of processes that vary over time [19] and IFT combined with fuzzy control [20].

We discussed in [21] the signal processing aspects of the IFT-based state feedback control for second-order positioning systems which have an integral component. A state-space formulation of IFT is analyzed in [22], and the solution converges to the analytical solutions for the state feedback gain matrix and to the Kalman gain. A Linear Quadratic Gaussian (LQG) formulation supported by the transfer function formulation, validated by digital simulation results for a first order process, is offered in [23]. Another LQG formulation dedicated to servo systems control with the Kalman filter state observer was validated in our recent paper [24].

This paper presents an extension of IFT for the optimal state feedback control techniques. Our state feedback CS estimates the OF gradients directly on the basis of measurements carried out during the CS operation. The accent is put on the interpretation of the results obtained in the particular case where a LQR-based tuning is attempted. An original IFT-based approach based on a data-based algorithm to improve the performance of state feedback control systems for single input processes is offered. A comparison between the model-based design for state feedback optimal control systems (the LQR problem) and the experimental-based design using IFT is carried out.

The LQR approach is applied in this paper to initially tune the parameters of the state feedback controller, and our approach ensures further improvement of the CS performance. This improvement is achieved by the alleviation of the OF using experiment-based information from the real-world CS. Our approach makes use of the LQR to guarantee that the initial controller is sufficiently close to the optimal one for the gradient scheme to converge. Our approach is appealing due to several situations that can occur in practice: differences between process models and reality, process changes in time and modifications of performance specifications.

This paper is structured as follows: the next section discusses the general framework to for tuning the state feedback CSs by means of IFT. Section 3 focuses on the new IFT algorithm. Section 4 is dedicated to the case study of an IFT-based angular position controller for a DC servo system with actuator dead zone and control signal saturation. Several practical recommendations for CS designers are also given. The conclusions are highlighted in Section 5. Appendix 1 
shows the connection between the LQR OF, which drives the analytical solutions of the optimisation problem, and the IFT OF, which is subjected to practical evaluations in our data-based algorithm.

\section{IFT of State Feedback Control Systems}

Let us consider a process characterized by the single input discrete-time linear time-invariant (LTI) state-space model

$$
\begin{aligned}
& \mathbf{x}(k+1)=\mathbf{A} \mathbf{x}(k)+\mathbf{B} u(k)+\overline{\mathbf{B}} \mathbf{w}(k), \\
& \mathbf{y}(k)=\mathbf{C} \mathbf{x}(k)+\overline{\mathbf{C}} \mathbf{v}(k),
\end{aligned}
$$

where $k \in \mathbf{N}$ is the discrete time argument, $u$ is the control signal, $\mathbf{x}=\left[\begin{array}{lll}x_{1} & \ldots & x_{n}\end{array}\right]^{T} \in \mathbf{R}^{n}$ is the state vector, $n$ is the system order, $\mathbf{y} \in \mathbf{R}^{n_{y}}$ is the controlled output, $\quad \mathbf{A} \in \mathbf{R}^{n \times n}, \quad \mathbf{B} \in \mathbf{R}^{n \times 1}, \quad \overline{\mathbf{B}} \in \mathbf{R}^{n \times n}, \quad \mathbf{C} \in \mathbf{R}^{n_{y} \times n}$, $\overline{\mathbf{C}} \in \mathbf{R}^{n_{y} \times n}$ are constant matrices, and $\mathbf{w} \in \mathbf{R}^{n}$ and $\mathbf{v} \in \mathbf{R}^{n_{y}}$ are the uncorrelated process noise vector and measurement noise vector, respectively. All elements of the vectors $\mathbf{w}$ and $\mathbf{v}$ are normal independent identically distributed random variables with zero means and variances $\sigma_{w}^{2}$ and $\sigma_{v}^{2}$, respectively. Zero initial conditions are assumed throughout the paper for the process dynamics without generality loss. The process is supposed to be controllable and observable.

The vector $\mathbf{y}$ is the controlled position and speed in the cases of positioning systems and of servo systems in several applications [25]-[32], but our approach is not limited to positioning systems, servo systems or mechatronics. The transfer characteristics of the actuator and of the measurement instrumentation of the state variables $x_{i}, i=1 \ldots n$, are both included in the process. The corresponding deterministic discrete-time LTI state-space model of the process is

$$
\begin{aligned}
& \mathbf{x}(k+1)=\mathbf{A} \mathbf{x}(k)+\mathbf{B} u(k), \\
& \mathbf{y}(k)=\mathbf{C} \mathbf{x}(k) .
\end{aligned}
$$

The following infinite horizon quadratic performance index can be imposed as performance specification of the CS such that its minimization can ensure very good CS performance:

$$
I(\boldsymbol{\rho})=\sum_{k=0}^{\infty}\left[\mathbf{x}^{T}(\boldsymbol{\rho}, k) \mathbf{Q} \mathbf{x}(\boldsymbol{\rho}, k)+\lambda u^{2}(\boldsymbol{\rho}, k)\right],
$$


where $\boldsymbol{\rho}=\left[\begin{array}{lll}\rho_{1} & \cdots & \rho_{n}\end{array}\right]^{T}$ is a parameter vector, the state vector and the control signal are parameterised by $\boldsymbol{\rho}$, and the weights are

$$
\mathbf{Q} \geq 0, \mathbf{Q}=\left[q_{i j}\right]_{i, j=1 \ldots n}, q_{i j}=q_{j i}, i, j=1 \ldots n, \lambda>0 .
$$

The parametric optimisation of the state feedback control systems can be formulated as the following optimisation problem of finding the optimal parameter vector $\boldsymbol{\rho}^{*}$ which corresponds to the optimal gain matrix $\left(\boldsymbol{\rho}^{*}\right)^{T}$ :

$$
\boldsymbol{\rho}^{*}=\arg \min _{\boldsymbol{\rho}} I(\boldsymbol{\rho}) \text {. }
$$

The solution to the discrete-time infinite horizon optimisation problem defined in (5) is the control law $u\left(\boldsymbol{\rho}^{*}, k\right)=-\left(\boldsymbol{\rho}^{*}\right)^{T} \mathbf{x}\left(\boldsymbol{\rho}^{*}, k\right)$ which together with (2) drives the state vector to zero under the CS's spectrum characterized by the system matrix $\mathbf{A}^{c l}=\mathbf{A}-\mathbf{B}\left(\boldsymbol{\rho}^{*}\right)^{T}$.

The reference inputs are commonly introduced for each state variable when it is needed to drive the state vector to a different point in the state space. The resulting state feedback controller is defined in terms of the control law

$$
\begin{aligned}
u(\boldsymbol{\rho}, k) & =\boldsymbol{\rho}^{T} \mathbf{e}(\boldsymbol{\rho}, k), \boldsymbol{\rho}^{T}=\left[\begin{array}{lll}
\rho_{1} & \ldots & \rho_{n}
\end{array}\right], \\
\mathbf{e}(\boldsymbol{\rho}, k) & =\mathbf{r}(k)-\mathbf{x}(\boldsymbol{\rho}, k),
\end{aligned}
$$

where $\mathbf{r}=\left[\begin{array}{lll}r_{1} & \ldots & r_{n}\end{array}\right]^{T}$ is the reference input vector, $r_{i}$ are the reference inputs that correspond to the state variables $x_{i}, i=1 \ldots n$, $\mathbf{e}=\left[\begin{array}{lll}e_{1}=r_{1}-x_{1} & \ldots & e_{n}=r_{n}-x_{n}\end{array}\right]^{T}$ is the state control error vector that consists of the state variable errors $e_{i}, i=1 \ldots n, \boldsymbol{\rho}^{T}$ is the state feedback gain matrix, referred to also as the gain matrix, $\boldsymbol{\rho}$ is the parameter vector, and $T$ indicates the matrix transposition. The vector $\mathbf{e}$ is applied as an input to the state feedback gain matrix $\boldsymbol{\rho}^{T}$ as shown in Figure 1, where $\mathbf{P}$ is the process and $\mathbf{C}$ is the controller, and the difference from the matrix $\mathbf{C}$ in (1) will be pointed out in the sequel when necessary.

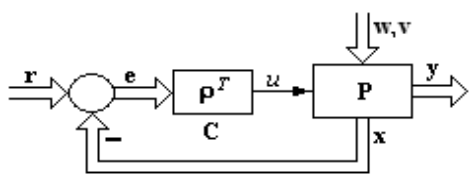

Figure 1

State feedback control system structure 
Introducing reference inputs for the state variables, the optimisation problem defined in (5) makes use of the following modified OF:

$I(\boldsymbol{\rho})=\sum_{k=0}^{\infty}\left[\mathbf{e}^{T}(\boldsymbol{\rho}, k) \mathbf{Q} \mathbf{e}(\boldsymbol{\rho}, k)+\lambda \mathbf{e}_{u}^{2}(\boldsymbol{\rho}, k)\right]$,

where the control signal error $e_{u}(\boldsymbol{\rho}, k)$ is defined as the difference between the control signal and its steady-state value $u(\boldsymbol{\rho}, \infty)$ :

$e_{u}(\boldsymbol{\rho}, k)=u(\boldsymbol{\rho}, k)-u(\boldsymbol{\rho}, \infty)$.

In order to apply the IFT to solve the optimisation problem (5), using the OF defined in (7), we will use a modified OF, referred to as $J$, defined as follows over the finite time horizon $N$ for reasons of practical evaluations of the OF:

$J(\boldsymbol{\rho})=\sum_{k=0}^{N}\left[\mathbf{e}^{T}(\boldsymbol{\rho}, k) \mathbf{Q} \mathbf{e}(\boldsymbol{\rho}, k)+\lambda e_{u}^{2}(\boldsymbol{\rho}, k)\right]$.

The OF (9) can be represented by the following approximation if $N$ is sufficiently large to capture all transients in the CS response:

$I(\boldsymbol{\rho}) \approx J(\boldsymbol{\rho})$.

IFT algorithms can conveniently be employed to find a solution $\boldsymbol{\rho}^{*}$ to the optimisation problem

$$
\boldsymbol{\rho}^{*}=\arg \min _{\boldsymbol{\rho} \in D_{\mathrm{S}}} J(\boldsymbol{\rho}),
$$

where $D_{S}$ stands for the stability domain of all state feedback gain matrixes that ensure a stable CS. The two optimisation problems defined in (5) and respectively in (11) essentially are equivalent. However, differences may appear due to the infinite and respectively the finite time horizons in the OFs and to the more general stochastic framework that is necessary to be taken into consideration when the IFT problem is set.

The finite time optimal state feedback control problem is characterized by a timevarying gain matrix, while the infinite time state feedback optimal control problem is characterized by a steady-state gain matrix $\boldsymbol{\rho}^{T}$. The calculation of the matrices used in both cases requires process models that are affected by modelling and identification errors. In order to solve the optimisation problem (11), a parameter vector $\boldsymbol{\rho}$ has to be found such that

$$
\frac{\partial J}{\partial \boldsymbol{\rho}}=\left[\begin{array}{lll}
\frac{\partial J}{\partial \rho_{1}} & \ldots & \frac{\partial J}{\partial \rho_{n}}
\end{array}\right]^{T}=\left[\begin{array}{lll}
0 & \ldots & 0
\end{array}\right]^{T},
$$


which, for an OF $J$ defined in (9), becomes

$$
\frac{\partial J}{\partial \rho_{l}}=2 \sum_{k=0}^{N}\left\{\left[\sum_{\substack{i, j=1 \\ i \geq j}}^{n}\left(q_{i j} e_{i} \frac{\partial e_{j}}{\partial \rho_{l}}\right)\right]+\lambda e_{u} \frac{\partial e_{u}}{\partial \rho_{l}}\right\}=0, l=1 \ldots n
$$

The cases of constrained optimisation problems use the Karush-Kuhn-Tucker optimality conditions instead of the null gradient given by (12).

Partial derivatives $\frac{\partial e_{i}}{\partial \rho_{l}}$ and $\frac{\partial e_{u}}{\partial \rho_{l}}$ need to be first calculated in order to obtain the derivatives $\frac{\partial J}{\partial \rho_{l}}, l=1 \ldots n$, in the gradient of the OF. We will present in the next section an experimental method developed to calculate these partial derivatives.

The IFT algorithms are presented as follows in the more general stochastic framework. Therefore the OF defined in (9) and evaluated on a finite-time horizon becomes a random variable and therefore should be defined as

$$
J(\boldsymbol{\rho})=E\left\{\sum_{k=0}^{N}\left[\mathbf{e}^{T}(\boldsymbol{\rho}, k) \mathbf{Q} \mathbf{e}(\boldsymbol{\rho}, k)+\lambda \mathbf{e}_{u}^{2}(\boldsymbol{\rho}, k)\right]\right\},
$$

where $E\{\}$ is the expectation with respect to the stochastic disturbances. However, the deterministic case results in the simplification of the IFT algorithms.

The IFT algorithms can solve the optimisation problem defined in (14) by using the Robbins-Monro stochastic approximation algorithm, which iteratively approaches a zero of a function without the need to know its complete expression. There is no need for evaluations of the OF, but its first and eventually second partial derivatives are important. This result holds not only for the tuning approach based on sensitivity functions, but also the stochastic convergence is ensured with useful consequences when dealing with real world processes. The parameter vector $\boldsymbol{\rho}$ values are iteratively updated according to the following equation:

$$
\boldsymbol{\rho}^{i+1}=\boldsymbol{\rho}^{i}-\gamma^{i}\left(\mathbf{R}^{i}\right)^{-1} \operatorname{est}\left[\frac{\partial J}{\partial \boldsymbol{\rho}}\left(\boldsymbol{\rho}^{i}\right)\right], \mathbf{R}^{i}>0,
$$

where $i \in \mathbf{N}$ is the current iteration/experiment index, $\gamma^{i}>0$ is the step size, $\operatorname{est}\left[\frac{\partial J}{\partial \boldsymbol{\rho}}\left(\boldsymbol{\rho}^{i}\right)\right]$ is the unbiased estimate of the gradient, and the regular matrix $\mathbf{R}^{i}$ can be the estimate of the Hessian matrix, the Gauss-Newton approximation of the Hessian, or the identity matrix in the case of less demanding and slower convergent computations. The step size sequence $\left\{\gamma^{i}\right\}_{i \in \mathbf{N}}$ should evolve in time such as to satisfy some bounds. With this regard the conditions to ensure the convergence of the stochastic algorithm are given in [10], [12], [22]. 


\section{Description and Implementation of IFT Algorithm}

LQR requires always a linearized model or a collection of local models of the process (e.g., in the gain scheduling approach) in order to calculate the optimal parameter vector $\boldsymbol{\rho}^{*}$ which corresponds to the optimal gain matrix $\left(\boldsymbol{\rho}^{*}\right)^{T}$. The identification problem itself is a rather complex undertaking in the case of MIMO systems, which requires a special design of the experiments.

On the other hand, the IFT-based approach does not need exact process models, and special gradient experiments can be conveniently designed to avoid abnormal operation regimes. The initial tuning of the gain matrix is not a problem in the case of the LQR-based approach. However, finding an initial stabilising controller without knowing the process is not a trivial task. Finally, the IFT can be used to fine tune controllers for nonlinear processes under constraints [16].

The IFT-based approach offers a notable degree of flexibility. The OF (11) is not only weighting the state variable errors and the control signal error associated with the LTI state-space model of the CS defined in (1), but it can weigh the reference model tracking error trajectories as well. As shown in [18], the IFT can be used as an alternative solution to the popular pole placement design of optimal state feedback controllers. However, the form in which it is used here is similar to the classical LQR optimisation problem.

As mentioned in the previous section, the main advantage of the IFT resides in its gradient computation algorithm together with the stochastic convergence result. The MIMO IFT-based approach is particularly well suited to solving the optimisation problem defined in (9). From (1) and (6), the LTI state feedback CS is characterized by

$$
\begin{aligned}
& \mathbf{x}(\boldsymbol{\rho}, k)=\mathbf{P}_{u \mathbf{x}}\left(q^{-1}\right) u(\boldsymbol{\rho}, k)+\mathbf{P}_{\mathbf{w} \mathbf{x}}\left(q^{-1}\right) \mathbf{w}(k), \\
& u(\boldsymbol{\rho}, k)=\boldsymbol{\rho}^{T} \underbrace{[\mathbf{r}(k)-\mathbf{x}(\boldsymbol{\rho}, k)],}_{\mathbf{e}(\boldsymbol{\rho}, k)}
\end{aligned}
$$

where $\mathbf{P}_{u \mathbf{x}}\left(q^{-1}\right) \in \mathbf{R}^{n \times 1}$ is the process pulse transfer matrix operator from the input $u$ to the state vector $\mathbf{x}, \mathbf{P}_{\mathbf{w} \mathbf{x}}\left(q^{-1}\right) \in \mathbf{R}^{n \times n}$ is the disturbance pulse transfer matrix operator from the process noise vector $\mathbf{v}$ to the state vector, and $\mathbf{w}, \mathbf{x}$ and $u$ are defined in accordance with (1). The dependence of the variables involved in (17) on $\boldsymbol{\rho}$ is underlined accordingly.

As suggested in (13), we need to calculate the derivatives $\frac{\partial e_{i}}{\partial \rho_{l}}$. Taking into account the state feedback control law defined in (6) and the fact that $\mathbf{r}$ does not depend on $\boldsymbol{\rho}$, the partial derivatives obtain the expressions 


$$
\begin{aligned}
& \frac{\partial e_{i}(\boldsymbol{\rho}, k)}{\partial \rho_{l}}=-\frac{\partial x_{i}(\boldsymbol{\rho}, k)}{\partial \rho_{l}}, \\
& \frac{\partial e_{u}(\boldsymbol{\rho}, k)}{\partial \rho_{l}}=\frac{\partial u(\boldsymbol{\rho}, k)}{\partial \rho_{l}}-\frac{\partial u(\boldsymbol{\rho}, \infty)}{\partial \rho_{l}}, i, l=1 \ldots n .
\end{aligned}
$$

The derivative of the CS state vector with respect to a certain process parameter $\rho_{l}, l=1 \ldots n$, can be expressed as

$$
\frac{\partial \mathbf{x}(\boldsymbol{\rho}, k)}{\partial \rho_{l}}=\mathbf{P}_{u \mathbf{x}}\left(q^{-1}\right) \frac{\partial \mathrm{u}(\boldsymbol{\rho}, k)}{\partial \rho_{l}} .
$$

Similarly, the derivative of the control signal in the state feedback control law expressed in (6) with respect to the same parameter $\rho_{l}, l=1 \ldots n$, is

$$
\frac{\partial u(\boldsymbol{\rho}, k)}{\partial \rho_{l}}=\frac{\partial \boldsymbol{\rho}^{T}}{\partial \rho_{l}} \mathbf{e}(\boldsymbol{\rho}, k)-\boldsymbol{\rho}^{T} \frac{\partial \mathbf{x}(\boldsymbol{\rho}, k)}{\partial \rho_{l}} .
$$

The derivative of the gain matrix $\boldsymbol{\rho}^{T}$ with respect to one parameter $\rho_{l}$ is a row vector with the same dimension as $\boldsymbol{\rho}^{T}$, but with a single nonzero element that takes the value 1 , and when multiplied by $\mathbf{e}$ it keeps only the $l^{\text {th }}$ state variable error. The derivative of the control signal is then

$$
\begin{aligned}
\frac{\partial u(\boldsymbol{\rho}, k)}{\partial \rho_{l}} & =\left[\begin{array}{lllll}
0 & \ldots & \rho_{l} & \ldots & 0
\end{array}\right]\left[\begin{array}{c}
e_{1}(\boldsymbol{\rho}, k) \\
\vdots \\
e_{l}(\boldsymbol{\rho}, k) \\
\vdots \\
e_{n}(\boldsymbol{\rho}, k)
\end{array}\right]-\boldsymbol{\rho}^{T} \frac{\partial \mathbf{x}(\boldsymbol{\rho}, k)}{\partial \rho_{l}} \\
& =e_{l}(\boldsymbol{\rho}, k)+\boldsymbol{\rho}^{T}\left(\underset{=\mathbf{0}}{\mathbf{r}}-\frac{\partial \mathbf{x}(\boldsymbol{\rho}, k)}{\partial \rho_{l}}\right),
\end{aligned}
$$

where $e_{l}$ is the $l^{\text {th }}$ state variable error. Equation (21) shows how to conduct the gradient experiments with the process: by injecting an additive term in the control signal of the state feedback CS and letting the reference input vector $\mathbf{r}$ equal to zero, the derivatives of the state variables and of the control signal with respect to the parameter $\rho_{l}$ in $\boldsymbol{\rho}^{T}$ are obtained. The injected term is $e_{l}$, i.e., the $l^{\text {th }}$ element of the state control error vector obtained in a normal experiment. All specific experiments of IFT are described as follows. 
An initial experiment, called the normal experiment, is carried out to record the evolution of the state variables and the corresponding state variable errors and control signal error respectively, in the state feedback CS shown in Figure 1.

Other $n$ gradient experiments are then subsequently carried out in order to calculate estimates of the derivatives $\frac{\partial x_{i}}{\partial \rho_{l}}$ and $\frac{\partial u}{\partial \rho_{l}}$, and use is made of (17) and

(21). Let $l$ denote as a superscript the $l^{\text {th }}$ gradient experiment corresponding to $\rho_{l}, l=1 \ldots n$ :

$$
\begin{aligned}
\mathbf{x}^{l}(\boldsymbol{\rho}, k) & =\mathbf{P}_{u \mathbf{x}}\left(q^{-1}\right) u^{l}(\boldsymbol{\rho}, k)+\mathbf{P}_{\mathbf{w} \mathbf{x}}\left(q^{-1}\right) \mathbf{w}^{l}(k) \\
& =\mathbf{P}_{u \mathbf{x}}\left(q^{-1}\right)\left[e_{l}(\boldsymbol{\rho}, k)-\boldsymbol{\rho}^{T} \mathbf{x}^{l}(\boldsymbol{\rho}, k)\right]+\mathbf{P}_{\mathbf{w} \mathbf{x}}\left(q^{-1}\right) \mathbf{w}^{l}(k) .
\end{aligned}
$$

Equation (22) provides the basis for the experimental setup (illustrated in Figure 2) employed in the iterative calculation of the partial derivatives $\frac{\partial x_{i}}{\partial \rho_{l}}$ and $\frac{\partial u}{\partial \rho_{l}}$ needed in the minimization of the OF. We actually obtain at each gradient experiment the estimates of the gradient of the state variables with respect to the gain matrix parameters. In other words, the state variables of the gradient experiments are actually the gradient estimates. This is because at each experiment the process noise acts upon the CS. Equation (22) results in $E\left\{\mathbf{x}^{l}\right\}=\frac{\partial \mathbf{x}}{\partial \rho_{l}}$.

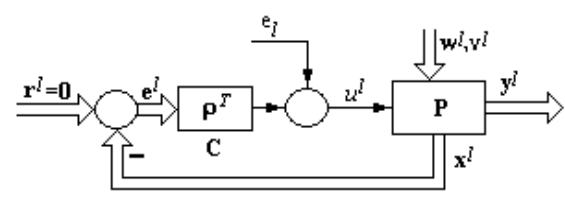

Figure 2

Experimental setup to compute $\frac{\partial x_{i}}{\partial \rho_{l}}$ and $\frac{\partial u}{\partial \rho_{l}}$

The IFT algorithm consists of the following steps:

- $\quad$ Step 0. Set the step size, the initial controller parameters $\boldsymbol{\rho}^{0}$ and the weights in the OF.

- Step 1. Conduct the initial (normal) experiment making use of the CS structure presented in Figure 1 and record the evolution of all state variables. 
- $\quad$ Step 2. Conduct the $n$ gradient experiments making use of the experimental setup presented in Figure 2 to obtain all partial derivatives $\frac{\partial x_{i}}{\partial \rho_{l}}$ and $\frac{\partial u}{\partial \rho_{l}}$.

- Step 3. Conduct the normal experiment again such that the states contain realizations of noise that differ from the noise at step 2 to ensure the unbiased estimate of the gradient.

- $\quad$ Step 4. Calculate the estimates of the gradient of the OF according to (13).

- $\quad$ Step 5. Compute $\boldsymbol{\rho}^{i+1}$ in terms of the update law (15).

Step 0 is done only once. Steps 1 to 5 are repeated iteratively. Step 0 requires an initial set of parameters that stabilise the state feedback CS to be obtained here by LQR. In the case of Single Input-Single Output (SISO) systems, we can use the Ziegler-Nichols tuning [33] or other techniques like the Virtual Reference Feedback Tuning [34], [35] in order to get these parameters.

There exists a difference between the deterministic case and the stochastic case in terms of the objective function and of the objectives that are targeted. Specifically, IFT is developed as an experimental-based technique in which the noise enters the $\mathrm{CS}$ and therefore the objective function also contains a factor that depends on the noise; therefore the minimization of the energy transfer between the noise and the state variables is also attempted, in addition to the minimization of the state control error and of the control signal energy that are objectives specific to the LQR deterministic problem. This aspect is illustrated in Appendix 1.

\section{Case Study}

The case study is a second-order positioning CS for a modular DC servo system with an integral component. The process is characterized by the single input discrete-time LTI state-space model defined in (4) with the matrices

$$
\mathbf{A}=\left[\begin{array}{ll}
1 & 0.0487 \\
0 & 0.9471
\end{array}\right], \mathbf{B}=\left[\begin{array}{l}
0.1867 \\
7.3993
\end{array}\right], \mathbf{C}=\mathbf{I}_{2},
$$

and with the angular position and the angular speed as state variables. The experimental setup is built around the INTECO DC servo system laboratory equipment. The control signal $u$ for the accepted laboratory equipment is the PWM duty-cycle constrained to $-1 \leq u \leq 1$. The actuator exhibits a \pm 0.15 width insensitivity zone applied to $u$ and compensated by an inverse nonlinearity. 
The simplified model (23) was obtained by the parameter identification of the first-principle model of the equipment resulting in the simplified process transfer function (considering $u$ as the input and the angular position as the output)

$$
P(s)=k_{P} /\left[s\left(1+T_{\Sigma} s\right)\right],
$$

where $k_{P}$ is the process gain and $T_{\Sigma}$ is the small time constant. The values of the process parameters were obtained as $k_{P}=139.88$ and $T_{\Sigma}=0.92 \mathrm{~s}$. Using the notation $T_{s}$ for the sampling period, the sampling period of $T_{s}=0.05 \mathrm{~s}$ was set.

The detailed mathematical model of the process is time variant due to the interchanging modules (inertial load, encoder and eventually backlash). The reidentification is not used in our approach. An experimental scenario is presented as follows to illustrate the benefits of the IFT-based approach over the classical LQR-based approach.

The weights $\mathbf{Q}$ and $\lambda$ in the infinite horizon quadratic performance index defined in (5) are

$$
\mathbf{Q}=\left[\begin{array}{cc}
0.2 & 0 \\
0 & 0.2
\end{array}\right], \lambda=400 \text {. }
$$

The results are presented for a step angular position reference input of $40 \mathrm{rad}$ and zero angular speed reference input, i.e., $\mathbf{r}=\left[\begin{array}{ll}40 & 0\end{array}\right]^{T}$. A first order low-pass digital filter with a cut-off frequency of $20 \mathrm{rad} / \mathrm{s}$ is used in the experiments to reduce the errors and the noise that occurs during the measurement of the angular speed. This filter will change the process model, but IFT is independent with this regard. This choice also supports the idea that the tuning can be carried out whenever the process model changes in time, without the need of identification and optimal redesign via $\mathrm{LQR}$.

The weights (25) do not cause the saturation of the actuator. Thus the undesired behaviour due to the nonlinearities is avoided. This undesired behaviour usually occurs in the LQR-based approach where the nonlinear actuator is not included in the process model.

For benchmarking purposes the control system performance indices that are used are the $\mathrm{OF}$, the control signal energy defined as

$$
E_{u}=\sum_{k=0}^{N} u^{2}(k),
$$

the $10 \%$ to $90 \%$ rise time of the position response $\left(t_{r}\right)$, and the maximum speed $\left(\omega_{\max }\right)$. The IFT-based approach is next used to further reduce the OF, taking advantage of the experiments conducted on the real-world experimental setup. 
In order to provide a relevant improvement, we start with a process model that is very different from the identified model. This is the same as assuming that the process model is time variant or that the identification is not accurate. The starting model for the LQR design uses the process parameters $k_{P}=180$ and $T_{\Sigma}=1.2 \mathrm{~s}$ in the transfer function (24). For the weights (25), the state feedback gain matrix is

$$
\left(\boldsymbol{\rho}^{T}\right)_{L Q R_{-} 1}=\left[\rho_{1}=0.020496 \rho_{2}=0.021368\right],
$$

The gain matrix $\left(\boldsymbol{\rho}^{T}\right)_{L Q R_{-} 1}$ is further tuned using our IFT algorithm. The initial step size in the IFT algorithm employed to minimize the OF (9) is set to the initial value $\gamma^{0}=2 \cdot 10^{-8}$, the values of the consequent step sizes are set in terms of (17), and $\mathbf{R}^{i}=\mathbf{I}_{2}$ is used.

The reduction of the value of the OF is emphasized to illustrate that our IFT algorithm ensures the performance improvement of the state feedback CS. The following expression of the gain matrix is obtained after 15 iterations:

$$
\left(\boldsymbol{\rho}^{T}\right)_{L Q R_{-} 2}=\left[\rho_{1}=0.018900 \quad \rho_{2}=0.017355\right] .
$$

The evolution of the OF with respect to the iteration number (i.e., during the tuning) is presented in Figure 3. The evolutions of the controller parameters (i.e., the elements of the gain matrix) versus the iteration number are presented in Figure 4. The time responses of the CS before and after the application of the IFT algorithm are presented in Figure 5.

Figure 4 illustrates that the OF is affected by random disturbances when it is evaluated on the real-world process. The values of the OF for the gain matrices defined in (27) and (28) are $J_{L Q R_{-} 1}=3821.89$ and $J_{L Q R_{-} 2}=3772.10$, respectively. The following performance indices were obtained:

- $\quad$ for the initial CS response (i.e., before IFT): $E_{u}=2.5482, t_{r}=2.94 \mathrm{~s}$, $\omega_{\max }=27.0847 \mathrm{rad} / \mathrm{s}$,

- for the final CS response (i.e., after IFT): $E_{u}=2.4654, t_{r}=2.53 \mathrm{~s}$, $\omega_{\max }=27.9519 \mathrm{rad} / \mathrm{s}$.

A discussion on these results follows. The relatively large number of iterations shown in this case indicates that the slow convergence is due to the fact that the steepest descent direction is used and due to the fact that we are close to the true local minimum, and therefore only modest but still quantifiable improvements are seen in the figures. In practical situations it suffices to do several experiments in order to improve the performance in terms of the OF. 


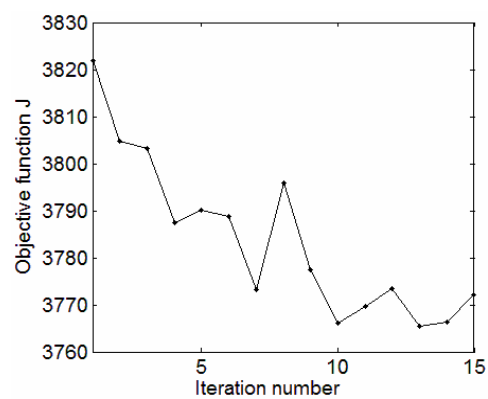

Figure 3

The evolution of the objective function versus the iteration number

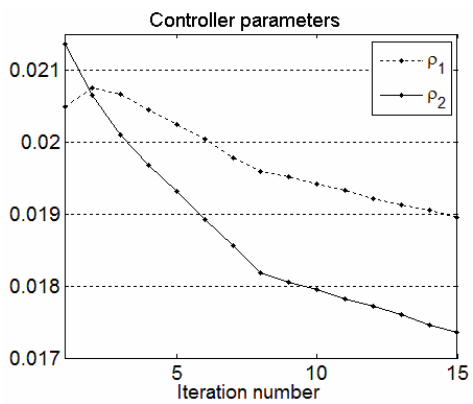

Figure 4

The evolution of the controller parameters versus the iteration number
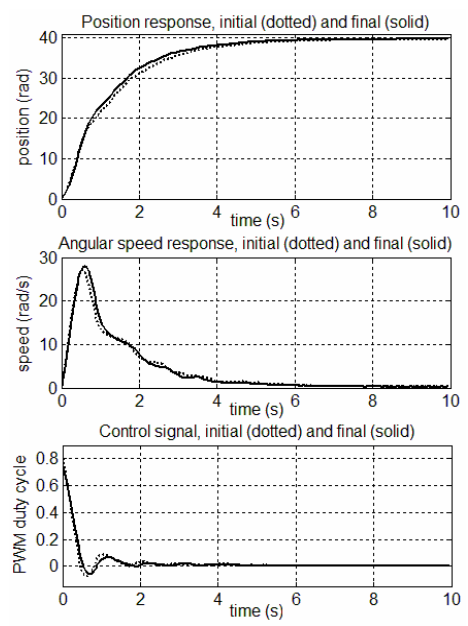

Figure 5

Control system responses of the CS before IFT and after IFT 
The minimization of $J$ is dedicated to reducing the energy transfer from the process noise to the state variables. In conclusion, nonzero reference inputs are reflected by targeting three objectives: the minimization of the tracking error energy, the minimization of the control effort, and the noise rejection problem [13]. The improvements via IFT shown in the previous section ensure the reduction of the OF value and of its variance, due to the lower sensitivity to noise. This idea is also backed up by Appendix 1 .

The time responses of the experimental results shown are not very different, and this shows the robustness of state feedback CS with respect to the controller and process parametric variations. However, the solution is an evident improvement of the LQR design, and when the noise contribution in the OF is small, it is expected that the tuning procedure gets near the optimal gain matrix, which results in an optimal state feedback CS with robustness properties. When the noise contribution is important, the robustness properties of the optimal state feedback CS still hold, as suggested by the simulation scenarios with included process noise.

The weights in the optimisation problems were set so as to ensure the linear operation of the process and of the actuator, viz., without entering saturation. The experimental results illustrate that the steady-state error of the position response is improved in spite of the process nonlinearities.

IFT requires $1+n$ real-time experiments per iteration, $n$ of them being successive gradient computation experiments. This number cannot be reduced using ideas similar to those presented in [36]-[38] because the number of gain matrix parameters is equal to the product between the numbers of process inputs and outputs.

\section{Conclusions}

This paper has presented an extension of the IFT approach to improve the performance of state feedback CSs where the performance specifications aim the minimization of OFs expressed as quadratic performance indices.

Our general IFT approach provides an efficient way to deal with some of the specific problems of ill-defined processes when the strongly model-dependent LQR design gives solutions that are far away from the optimal solution. In such cases, when the LQR approach cannot anymore allow finding the minimum of the $\mathrm{OF}$, the IFT approach can be applied to further reduce the OF. The experimental results presented in Section 4 show that the IFT approach, which allows an estimation the OF gradients on the basis of sensitivity functions and of real-time measurements during the CS operation, can successfully be used.

A limitation of our IFT approach is that it actually ensures the strong improvement of the CS performance and the strong reduction of the OF only with respect to the considered particular reference input. Modifications of the reference input will yield different results with different dynamic characteristics. Our IFT approach does not use state estimators, being developed for a specific situation where all 
states are measured. However, the introduction of state estimators in future research is not problematic because the estimator gain can also be included in the IFT algorithm.

Future research will deal with the extension of the proposed IFT approach to MIMO control systems in mechatronics applications and to the tuning of state feedback fuzzy control systems. Further study of the convergence of the IFT algorithms is needed for all these nonlinear applications. Similar model-free tuning methods will be implemented including extremum seeking with emphasis on the direct application to the tuning of fuzzy controllers [39]-[42].

\section{Acknowledgement}

This work was supported by a grant of the Romanian National Authority for Scientific Research, CNCS - UEFISCDI, project number PN-II-ID-PCE-2011-30109, and by a grant from the NSERC of Canada.

\section{References}

[1] I. Škrjanc, S. Blažič, O. E. Agamennoni: Identification of Dynamical Systems with a Robust Interval Fuzzy Model, Automatica, Vol. 41, No. 2, 2005, pp. 327-332

[2] M. A. Khanesar, M. Teshnehlab, O. Kaynak: Model Reference Fuzzy Control of Nonlinear Dynamical Systems Using an Optimal Observer, Acta Polytechnica Hungarica, Vol. 8, No. 4, 2011, pp. 35-54

[3] L. Kovács, B. Benyó, J. Bokor, Z. Benyó: Induced L L $_{2}$-Norm Minimization of Glucose-Insulin System for Type I Diabetic Patients, Computer Methods and Programs in Biomedicine, Vol. 102, No. 2, 2011, pp. 105-118

[4] E. S. Nicoară, F.-G. Filip, N. Paraschiv: Simulation-based Optimization Using Genetic Algorithms for Multi-objective Flexible JSSP, Studies in Informatics and Control, Vol. 20, No. 4, 2011, pp. 333-344

[5] S. Aykut, A. Kentli, S. Gülmez, O. Yazicioglu: Robust Multiobjective Optimization of Cutting Parameters in Face Milling, Acta Polytechnica Hungarica, Vol. 9, No. 4, 2012, pp. 85-100

[6] E. D. Niño: SAMODS and SAGAMODS: Novel Algorithms Based on the Automata Theory for the Multiobjective Optimization of Combinatorial Problems, International Journal of Artificial Intelligence, Vol. 8, No. S12, 2012, pp. 147-165

[7] P. R. Srivastava, M. Chis, S. Deb, X.-S. Yang: An Efficient Optimization Algorithm for Structural Software Testing, International Journal of Artificial Intelligence, Vol. 8, No. S12, 2012, pp. 68-77

[8] J. Vaščák, M. Pal'a: Adaptation of Fuzzy Cognitive Maps for Navigation Purposes by Migration Algorithms, International Journal of Artificial Intelligence, Vol. 8, No. S12, 2012, pp. 20-37 
[9] F. Yao, Z. Dong, K. Meng, Z. Xu, H. Iu, K. Wong: Quantum-inspired Particle Swarm Optimization for Power System Operations Considering Wind Power Uncertainty and Carbon Tax in Australia, IEEE Transactions on Industrial Informatics, Vol. 8, No. 4, 2012, pp. 880-888

[10] H. Hjalmarsson, M. Gevers, S. Gunnarsson: A Convergent Iterative Restricted Complexity Control Design Scheme, Proceedings of $33^{\text {rd }}$ IEEE Conference on Decision and Control, Lake Buena Vista, FL, USA, 1994, pp. $1735-1740$

[11] H. Hjalmarsson, M. Gevers, S. Gunnarsson, O. Lequin: Iterative Feedback Tuning: Theory and Applications, IEEE Control Systems Magazine, Vol. 18 , No. 4, 1998, pp. 26-41

[12] H. Hjalmarsson: Iterative Feedback Tuning - An Overview, International Journal of Adaptive Control and Signal Processing, Vol. 16, No. 5, 2002, pp. 373-395

[13] J. K. Huusom, N. K. Poulsen, S. B. Jørgensen: Data Driven Tuning of State Space Control Loops with Unknown State Information and Model Uncertainty, Computer Aided Chemical Engineering, Vol. 26, 2009, pp. $441-446$

[14] R.-E. Precup, S. Preitl, I. J. Rudas, M. L. Tomescu, J. K. Tar: Design and Experiments for a Class of Fuzzy Controlled Servo Systems, IEEE/ASME Transactions on Mechatronics, Vol. 13, No. 1, 2008, pp. 22-35

[15] S. Kissling, P. Blanc, P. Myszkorowski, I. Vaclavik: Application of Iterative Feedback Tuning (IFT) to Speed and Position Control of a Servo Drive, Control Engineering Practice, Vol. 17, No. 7, 2009, pp. 834-840

[16] H. Hjalmarsson: Control of Nonlinear Systems Using Iterative Feedback Tuning, Proceedings of 1998 American Control Conference (ACC 1998), Philadelphia, PA, USA, 1998, Vol. 4, pp. 2083-2087

[17] J. Sjöberg, F. De Bruyne, M. Agarwal, B. D. O. Anderson, M. Gevers, F. J. Kraus, N. Linard: Iterative Controller Optimization for Nonlinear Systems, Control Engineering Practice, Vol. 11, No. 9, 2003, pp. 1079-1086

[18] A. J. McDaid, K. C. Aw, S. Q. Xie, E. Haemmerle: Gain Scheduled Control of IPMC Actuators With 'Model-free' Iterative Feedback Tuning, Sensors and Actuators A: Physical, Vol. 164, No. 1-2, 2010, pp. 137-147

[19] A. J. McDaid, K. C. Aw, E. Haemmerle, S. Q. Xie: Control of IPMC Actuators for Microfluidics with Adaptive "Online" Iterative Feedback Tuning, IEEE/ASME Transactions on Mechatronics, Vol. 17, No. 4, 2012, pp. 789-797

[20] R.-E. Precup, M.-B. Rădac, M. L. Tomescu, E. M. Petriu, S. Preitl: Stable and Convergent Iterative Feedback Tuning of Fuzzy Controllers for 
Discrete-Time SISO Systems, Expert Systems with Applications, Vol. 40, No. 1, 2013, pp. 188-199

[21] M.-B. Rădac, R.-E. Precup, S. Preitl, E. M. Petriu, C.-A. Dragoş, A. S. Paul, S. Kilyeni: Signal Processing Aspects in State Feedback Control Based on Iterative Feedback Tuning, Proceedings of $2^{\text {nd }}$ International Conference on Human System Interaction (HSI '09), Catania, Italy, 2009, pp. $40-45$

[22] J. K. Huusom, N. K. Poulsen, S. B. Jørgensen: Data Driven Tuning of State Space Controllers with Observers, Proceedings of European Control Conference 2009 (ECC ’09), Budapest, Hungary, 2009, pp. 1961-1966

[23] J. K. Huusom, N. K. Poulsen, S. B. Jørgensen: Iterative Feedback Tuning of Uncertain State Space Systems, Brazilian Journal of Chemical Engineering, Vol. 27, No. 3, 2010, pp. 461-472

[24] M.-B. Rădac, R.-E. Precup, E. M. Petriu, S. Preitl: Application of IFT and SPSA to Servo System Control, IEEE Transactions on Neural Networks, Vol. 22, No. 12, 2011, pp. 2363-2375

[25] L. Horváth, I. J. Rudas: Modeling and Problem Solving Methods for Engineers, Academic Press, Elsevier, Burlington, MA: 2004

[26] J. A. Iglesias, P. Angelov, A. Ledezma, A. Sanchis: Evolving Classification of Agents' Behaviors: A General Approach, Evolving Systems, Vol. 1, No. 3, 2010, pp. 161-171

[27] Zs. Cs. Johanyák: Survey on Five Fuzzy Inference-based Student Evaluation Methods, in: Computational Intelligence in Engineering, I. J. Rudas, J. Fodor, J. Kacprzyk (Eds.), Springer-Verlag, Berlin, Heidelberg, New York, 2010, pp. 219-228

[28] D. Antić, S. Nikolić, M. Milojković, N. Danković, Z. Jovanović, S. Perić: Sensitivity Analysis of Imperfect Systems Using Almost Orthogonal Filters, Acta Polytechnica Hungarica, Vol. 8, No. 6, 2011, pp. 79-94

[29] O. Linda, M. Manic: Uncertainty-Robust Design of Interval Type-2 Fuzzy Logic Controller for Delta Parallel Robot, IEEE Transactions on Industrial Informatics, Vol. 7, No. 11, 2011, pp. 661-670

[30] P. Baranyi, Á. Csapó: Definition and Synergies of Cognitive Infocommunications, Acta Polytechnica Hungarica, Vol. 9, No. 1, 2012, pp. $67-83$

[31] H.-N. Teodorescu: Taylor and Bi-local Piecewise Approximations with Neuro-Fuzzy Systems, Studies in Informatics and Control, Vol. 21, No. 4, 2012, pp. 367-376

[32] M. Iwasaki, K. Seki, Y. Maeda: High-Precision Motion Control Techniques: A Promising Approach to Improving Motion Performance, IEEE Industrial Electronics Magazine, Vol. 6, No. 1, 2012, pp. 32-40 
[33] S. Kissling, P. Blanc, P. Myszkorowski, I. Vaclavik: Application of Iterative Feedback Tuning (IFT) to Speed and Position Control of a Servo Drive, Control Engineering Practice, Vol. 17, No. 7, 2009, pp. 834-840

[34] F. Previdi, T. Schauer, S. M. Savaresi, K. J. Hunt: Data-driven Control Design for Neuroprotheses: A Virtual Reference Feedback Tuning (VRFT) Approach, IEEE Transactions on Control Systems Technology, Vol. 12, No. 1, 2004, pp. 176-182

[35] M. C. Campi, S. M. Savaresi: Direct Nonlinear Control Design: The Virtual Reference Feedback Tuning (VRFT) Approach, IEEE Transactions on Automatic Control, Vol. 51, No. 1, 2006, pp. 14-27

[36] H. Hjalmarsson: Iterative Feedback Tuning of Linear Time-invariant MIMO Systems, Proceedings of $37^{\text {th }}$ IEEE Conference on Decision and Control, Tampa, FL, USA, 1998, pp. 3893-3898

[37] H. Hjalmarsson: Efficient Tuning of Linear Multivariable Controllers Using Iterative Feedback Tuning, International Journal of Adaptive Control and Signal Processing, Vol. 13, No. 7, 1999, pp. 553-572

[38] H. Jansson, H. Hjalmarsson: Gradient Approximations in Iterative Feedback Tuning for Multivariable Processes, International Journal of Adaptive Control and Signal Processing, Vol. 18, No. 8, 2004, pp. 665-681

[39] R.-E. Precup, S. Preitl: Fuzzy Controllers, Editura Orizonturi Universitare Publishers, Timisoara: 1999

[40] R. E. Precup, S. Doboli, S. Preitl: Stability Analysis and Development of a Class of Fuzzy Control Systems, Engineering Applications of Artificial Intelligence, Vol. 13, No. 3, 2000, pp. 237-247

[41] R.-E. Precup, S. Preitl, G. Faur: PI Predictive Fuzzy Controllers for Electrical Drive Speed Control: Methods and Software for Stable Development, Computers in Industry, Vol. 52, No. 3, 2003, pp. 253-270

[42] R.-E. Precup, S. Preitl, E. M. Petriu, J. K. Tar, M. L. Tomescu, C. Pozna: Generic Two-Degree-of-Freedom Linear and Fuzzy Controllers for Integral Processes, Journal of The Franklin Institute, Vol. 346, No. 10, 2009, pp. 980-1003

[43] A. S. Bazanella, M. Gevers, L. Mišković, B. D. O. Anderson: Iterative Minimization of $\mathrm{H}_{2}$ Control Performance Criteria, Automatica, Vol. 44, No. 10, 2008, pp. 2549-2559

\section{Appendix 1. Connection between LQR and IFT objective functions}

This Appendix illustrates the connection between the LQR OF which drives the analytical solutions of the optimisation problem, and the IFT OF which is subjected to practical evaluations in our data-based algorithm. We assume two 
cases for the OF, defined in the deterministic case and in the stochastic case related to the state feedback CS. The dependence on the parameter vector $\boldsymbol{\rho}$ is omitted for the sake of simplicity. Our development follows a similar development to that presented in [43], and the two cases, a) and b), are presented as follows.

a) The deterministic case. We assume that the following operational relationships are valid:

$$
\begin{aligned}
& \mathbf{x}(\boldsymbol{\rho}, k)=\mathbf{P}_{\mathbf{r} \mathbf{x}}\left(\boldsymbol{\rho}, q^{-1}\right) \mathbf{r}(k), u(\boldsymbol{\rho}, k)=\mathbf{P}_{\mathbf{r} u}\left(\boldsymbol{\rho}, q^{-1}\right) \mathbf{r}(k), \\
& \mathbf{e}(\boldsymbol{\rho}, k)=\mathbf{r}(k)-\mathbf{x}(\boldsymbol{\rho}, k)=\mathbf{r}(k)-\mathbf{P}_{\mathbf{r} \mathbf{x}}\left(\boldsymbol{\rho}, q^{-1}\right) \mathbf{r}(k),
\end{aligned}
$$

where $\mathbf{P}_{\mathbf{r x}}\left(\boldsymbol{\rho}, q^{-1}\right) \in \mathbf{R}^{n \times n}$ is the process pulse transfer matrix operator from the reference input vector $\mathbf{r}$ to the state vector $\mathbf{x}$ and $\mathbf{P}_{\mathbf{r} u}\left(\boldsymbol{\rho}, q^{-1}\right) \in \mathbf{R}^{n \times 1}$ is the process pulse transfer matrix operator from $\mathbf{r}$ to the control signal $u$. The dependence on $\boldsymbol{\rho}$ is assumed but not explicitly written as follows in order to simplify notation.

The infinite horizon OF specific to the formulation of the LQR problem corresponding to this case is

$$
\begin{aligned}
& I(\boldsymbol{\rho})=\sum_{k=0}^{\infty}\left\{\mathbf{e}(k)^{T} \mathbf{Q} \mathbf{e}(k)+\lambda u^{2}(k)\right\}=\sum_{k=0}^{\infty}\{[\mathbf{r}(k) \\
& \left.\left.-\mathbf{Q}\left[\mathbf{r}(k) \mathbf{P}_{\mathbf{r} \mathbf{x}}\left(q^{-1}\right) \mathbf{r}(k)\right]^{T}-\mathbf{P}_{\mathbf{r} \mathbf{x}}\left(q^{-1}\right) \mathbf{r}(k)\right]+\lambda\left[\mathbf{P}_{\mathbf{r} u}\left(q^{-1}\right) \mathbf{r}(k)\right]^{2}\right\} .
\end{aligned}
$$

b) The stochastic case. The following relations hold:

$$
\begin{aligned}
& \mathbf{x}(k)=\mathbf{P}_{\mathbf{r} \mathbf{x}}\left(q^{-1}\right) \mathbf{r}(k)+\mathbf{P}_{\mathbf{w} \mathbf{x}}\left(q^{-1}\right) \mathbf{w}(k), \\
& u(k)=\mathbf{P}_{\mathbf{r} u}\left(q^{-1}\right) \mathbf{r}(k)+\mathbf{P}_{\mathbf{w} u}\left(q^{-1}\right) \mathbf{w}(k), \\
& \mathbf{e}(k)=\mathbf{r}(k)-\mathbf{x}(k)=\mathbf{r}(k)-\mathbf{P}_{\mathbf{r} \mathbf{x}}\left(q^{-1}\right) \mathbf{r}(k)-\mathbf{P}_{\mathbf{w} \mathbf{x}}\left(q^{-1}\right) \mathbf{w}(k) .
\end{aligned}
$$

The reference input vector and the process noise are assumed to be quasistationary and uncorrelated, i.e.,

$$
E\left\{\mathbf{r}(k) \mathbf{w}^{T}(k)\right\}=\mathbf{0} .
$$

The expression of the OF used in IFT in this case is

$$
\begin{aligned}
& J(\boldsymbol{\rho})=E\left\{\sum_{k=0}^{\infty} \mathbf{e}(k)^{T} \mathbf{Q} \mathbf{e}(k)+\lambda u^{2}(k)\right\}=E\left\{\sum_{k=0}^{\infty}[\mathbf{r}(k)\right. \\
& \left.-\mathbf{P}_{\mathbf{r} \mathbf{x}}\left(q^{-1}\right) \mathbf{r}(k)-\mathbf{P}_{\mathbf{w} \mathbf{x}}\left(q^{-1}\right) \mathbf{w}(k)\right]^{T} \mathbf{Q}\left[\mathbf{r}(k)-\mathbf{P}_{\mathbf{r} \mathbf{x}}\left(q^{-1}\right) \mathbf{r}(k)\right. \\
& \left.\left.-\mathbf{P}_{\mathbf{w} \mathbf{x}}\left(q^{-1}\right) \mathbf{w}(k)\right]+\lambda\left[\mathbf{P}_{\mathbf{r} u}\left(q^{-1}\right) \mathbf{r}(k)+\mathbf{P}_{\mathbf{w} u}\left(q^{-1}\right) \mathbf{w}(k)\right]^{2}\right\},
\end{aligned}
$$




$$
\begin{aligned}
& J(\boldsymbol{\rho})=\sum_{k=0}^{\infty} E\left\{\left[\mathbf{r}(k)-\mathbf{P}_{\mathbf{r} \mathbf{x}}\left(q^{-1}\right) \mathbf{r}(k)\right]^{T} \mathbf{Q}\left[\mathbf{r}(k)-\mathbf{P}_{\mathbf{r} \mathbf{x}}\left(q^{-1}\right) \mathbf{r}(k)\right]\right\} \\
& -\sum_{k=0}^{\infty} E\left\{\left[\mathbf{r}(k)-\mathbf{P}_{\mathbf{r} \mathbf{x}}\left(q^{-1}\right) \mathbf{r}(k)\right]^{T} \mathbf{Q} \mathbf{P}_{\mathbf{w} \mathbf{x}}\left(q^{-1}\right) \mathbf{w}(k)\right\} \\
& -\sum_{k=0}^{\infty} E\left\{\left[\mathbf{P}_{\mathbf{w} \mathbf{x}}\left(q^{-1}\right) \mathbf{w}(k)\right]^{T} \mathbf{Q}\left[\mathbf{r}(k)-\mathbf{P}_{\mathbf{r} \mathbf{x}}\left(q^{-1}\right) \mathbf{r}(k)\right]\right\} \\
& +\sum_{k=0}^{\infty} E\left\{\left[\mathbf{P}_{\mathbf{w} \mathbf{x}}\left(q^{-1}\right) \mathbf{w}(k)\right]^{T} \mathbf{Q}\left[\mathbf{P}_{\mathbf{w} \mathbf{x}}\left(q^{-1}\right) \mathbf{w}(k)\right]\right\} \\
& +\lambda \sum_{k=0}^{\infty} E\left\{\left[\mathbf{P}_{\mathbf{r} u}\left(q^{-1}\right) \mathbf{r}(k)\right]^{2}\right\}+ \\
& +2 \lambda \sum_{k=0}^{\infty} E\left\{\left[\mathbf{P}_{\mathbf{r} u}\left(q^{-1}\right) \mathbf{r}(k)\right]\left[\mathbf{P}_{\mathbf{w} u}\left(q^{-1}\right) \mathbf{w}(k)\right]\right\} \\
& +\lambda \sum_{k=0}^{\infty} E\left\{\left[\mathbf{P}_{\mathbf{w} u}\left(q^{-1}\right) \mathbf{w}(k)\right]^{2}\right\} .
\end{aligned}
$$

The second, the third and the sixth terms in (34) are zero due to the uncorrelation between $\mathbf{r}$ and $\mathbf{w}$. Therefore the following expression of $J(\boldsymbol{\rho})$ is obtained:

$$
J(\boldsymbol{\rho})=I(\boldsymbol{\rho})+\underbrace{}_{J_{\mathbf{w}}(\boldsymbol{\rho})} \underbrace{\sum_{k=0}^{\infty} E\left\{\left[\mathbf{P}_{w x}\left(q^{-1}\right) \mathbf{w}(k)\right]^{T} \mathbf{Q}\left[\mathbf{P}_{w x}\left(q^{-1}\right) \mathbf{w}(k)\right]\right\}} .
$$

The term $J_{\mathbf{w}}(\boldsymbol{\rho})$ is dedicated to the minimization of the energy transfer from $\mathbf{w}$ to $\mathbf{x}$ and to $u$. Inherently, in experiment-based tuning via IFT, this objective is also targeted in addition to the objectives to minimize the state control error energy (set-point tracking) and $E_{u}$. If $\mathbf{r}=\mathbf{0}$ and $\lambda=0$ are chosen, the OF $J(\boldsymbol{\rho})$ enables the minimization of the energy transfer from the process noise to the state variables, resulting in a non-robust structure. 